\title{
IMPAIRED OBJECT RECOGNITION MEMORY AND ACETYLCHOLINESTERASE ACTIVITY IN ANIMAL MODEL OF POST-TRAUMATIC STRESS DISORDER-RESTORED BY ECLIPTA ALBA LINN. A DIETARY HERB.
}

\author{
VIDYASHREE HM, MALATHI S, RAVINDRAN R
}

Department of Physiology, Dr. ALM PG Institute of Basic Medical Sciences, University of Madras, Chennai - 600 113, Tamil Nadu, India. Email: drravindranrajan@gmail.com

Received: 19 July 2016, Revised and Accepted: 30 August 2016

\section{ABSTRACT}

Objective: Acetylcholinesterase (AChE) inhibitors are important therapeutic targets to treat memory impairment caused due to stress, post-traumatic stress disorder (PTSD) results from traumatic stress exposure. Cognition and mood symptoms can begin or worsen after the traumatic event. In this study, we aimed to evaluate the role of Eclipta alba Linn. as an antistressor, AChE inhibitor, and on object recognition memory in an animal model of PTSD.

Methods: Adult male Wistar albino rats were randomly divided into four groups: Control, chronic unpredictable stress (CUS) (30 days), CUS+ethanolic extract of E. alba (EEEA) (200 mg/kg body weight), and EEEA (200 mg/kg body weight) treatment groups and were assessed for novel object recognition task (NORT), plasma corticosterone, and AChE activity.

Results: We found significant improvement in NORT $(\mathrm{p}<0.05)$ plasma corticosterone levels in stress group was significantly increased (p<00.05) which is resumed with EEEA treatment $(\mathrm{p}<0.05) \mathrm{AChE}$ activity was found to be reduced after EEEA treatment.

Conclusion: EEEA is found to possess cognitive enhancing activity in the animal model of PTSD.

Keywords: Recognition memory,PTSD,Unpredictable stress,Eclipta alba

(C) 2016 The Authors. Published by Innovare Academic Sciences Pvt Ltd. This is an open access article under the CC BY license (http://creativecommons. org/licenses/by/4. 0/) DOI: http://dx.doi.org/10.22159/ajpcr.2016.v9s3.14190

\section{INTRODUCTION}

Stress is an inevitable part of life, the relationship between stress and cognition is complex, and cognitive impairment due to stress depends on the type, duration, and severity. Stress is increasingly being recognized as the precipitant of several psychiatric illnesses including anxiety and depression [1]. Chronic variable stress models have been proven to be more useful as they are devoid of the problem of resistance in the animal species toward the commonly used stressors and also have the advantage of the development of effective and long-term stress response. Thus, chronic unpredictable stress (CUS) models are nowadays the preferred models for the generation of a stress response [2]. Chronic variable or unpredictable stress has shown to be consistent with the constellation of symptoms associated with post-traumatic stress syndrome, such as re-experiencing, and arousal to fearful contexts, validating CUS as a model for posttraumatic stress disorder (PTSD) in animals [3]. Different types of stressors such as noise stress, psychological stressors have proven to alter neurochemical, neurobehavioral as well as neurotransmitters in different brain regions [4-6] and also individuals with PTSD shown to have smaller hippocampal volumes [7-9] and exhibit impaired performance on hippocampal-dependent tasks $[10,11]$. Stress affects long-term potentiation and epigenetically regulates the hypothalamicpituitary-adrenal axis [12]. Recognition memory refers to the ability to judge a previously encountered item as familiar and depends on the integrity of the medial temporal lobe [13]. Investigations of the neural basis of recognition memory have implicated several brain regions. Recognition memory involving judgment of prior occurrence for individual items, relies on the perirhinal cortex, whereas recognition memory that involves multiple items and their contextual associations or the temporal order, in which items are encountered depends on interactions between the perirhinal cortex, hippocampus, and medial prefrontal cortex [14]. Recognition memory is strongly correlated with hippocampal and cortical functional integrity in both rodents' and primates' brains [15]. Moreover, both cortical and hippocampal dysfunctions affect novel object recognition (NOR) paradigm results [16]. Acetylcholine (ACh) is a neurotransmitter involved in memory and learning processing in the cholinergic system. Acetylcholinesterase (AChE) is the enzyme that decreases ACh levels by hydrolysis. The high AChE activity is present in the chronically stressed brain [6]. Eclipta alba Linn. (Family-Compositae) commonly called as Bhringaraj grows widely as an annual weed in moist places and is widely used in traditional system of medicine and is reported to possess antihyperglycemic, antihepatotoxic, antioxidant, antiinflammatory, antiaggressive, antifungal activities, and also a good hair growth regulator [17]. Phytochemically, E. alba is rich in wedelolactone, $\beta$-amyrin, stigmasterol, and luteolin-7-glucoside [18,19]. Previous investigations have revealed that $E$. alba herbal supplementation can significantly control cognitive and motor neuron dysfunction in old age. Similarly, medicinal plants and their bioactive constituents can improve behavioral (motor and cognitive behavior), neuronal signaling, and antiinflammatory effects [20]. Different extracts and its fractions of E. alba were analyzed for their antioxidant, AChE inhibitory, and antifungal activity, which have shown the best antioxidant capacities also have the most potential inhibitors of AChE [21]; hence, the present study was undertaken to evaluate the effect ethanolic extract of $E$. alba (EEEA) on AChE activity and object recognition memory in animal model of PTSD.

\section{METHODS}

\section{Experimental animals}

Male Wistar rats, weighing 200-225 g (2-2.5 months old) obtained from the Central Animal House Facility from Dr. ALM PG Institute of Basic Medical Sciences, were used for the experiments. The rats were housed and maintained in standard laboratory conditions. Food and water were provided ad-libitum unless specified otherwise in stress protocols. The experimental protocols were approved by the Institutional Animal Ethics Committee (IAEC No-01/25/2014). All efforts were made to minimize both the suffering and the number of animals used. Rats were randomly divided into IV groups consisting 
of six rats each: Group I served as normal control; Group II animals were induced with CUS (Table 1); Group III animals were pretreated for 15 days with $200 \mathrm{mg} / \mathrm{kg}$ bw EEEA followed by 30 days CUS+EEEA treatment; IV group served as treatment group where animals were treated with $200 \mathrm{mg} / \mathrm{kg}$ body weight EEEA for 45 days.

Induction of stress [22] (Modifiedkatz et al., 1982)

Single stressor per day was given in an unpredictable manner with no single stressor repeating on consecutive days (Table 1).

\section{Drug treatment}

The dried whole plant of E. alba Linn. was collected from Amruth Kesari Depot, Bangalore-53). Identification and authentication was done by Dr. Aravind, Assistant Professor, Department of Medical Botany, National Institute of Siddha (Reg. No of the certificate: NIS/MB/92/2013). The whole plant was coarsely powdered and the extraction was done in succession using Soxhlet extraction method. All the extracts were made solvent free and concentrated using rotary evaporator and preserved at $4^{\circ} \mathrm{C}$ in the airtight bottle until further use.

\section{Dosage and route of administration}

EEEA was dissolved in warm normal saline $(0.9 \% \mathrm{NaCl})$ dose $-200 \mathrm{mg} / \mathrm{kg}$ body weight [23] was administered through the oral route. Drug was administered $1 \mathrm{hr}$ before induction of stress in case of stress+treatment group.

\section{Plasma corticosterone assay}

Assay of corticosterone [24] is based on the oxidation of corticosteroids with ferric iron (III) in acidic medium and subsequent complex with ferrous iron (II) and potassium hexacyanoferrate. $0.5 \mu \mathrm{l}$ was mixed with appropriate volumes of the working solutions of corticosterone and was transferred into a series of $10 \mathrm{ml}$ volumetric asks. $2 \mathrm{ml}$ of sulfuric acid and $2 \mathrm{ml}$ of ferric chloride were added to $0.5 \mathrm{ml}$ of potassium hexacyanoferrate (III) solution. This mixture was heated in a water bath maintained at $70^{\circ} \mathrm{C} \pm 2^{\circ} \mathrm{C}$ for 30 minutes with occasional shaking and diluted to the $5 \mathrm{ml}$ mark with distilled water. Absorbance was measured at $780 \mathrm{~nm}$ against the reagent blank.

\section{Assay of AChE [24]}

AChE activity was measured by modified Ellman's method (Ellman et al., 1961). At the end of experimentation, rats were decapitated; the frontal cortex, hippocampus, and striatum were dissected quickly. The tissues were homogenized in $0.1 \mathrm{M}$ phosphate buffer, $\mathrm{pH} 8.0$ The reaction mixture consisted of $2.6 \mathrm{~mL}$ of phosphate buffer $(0.1 \mathrm{M}$, $\mathrm{pH}$ 8.0), $0.4 \mathrm{~mL}$ aliquot of homogenate, and $0.1 \mathrm{~mL}$ of $0.01 \mathrm{M}$ dithiobis nitrobenzoic acid. After addition of the substrate, acetylthiocholine iodide $(0.075 \mathrm{M})$, the change in absorbance was noted every 2 minutes for 10 minutes at $412 \mathrm{~nm}$ using an LKB spectrophotometer. The activity was expressed as micromoles hydrolyzed per minute per gram of tissue.

\section{NOR task (NORT) [25]}

NORT is a model of recognition memory and is based on the innate behavior of animals to spend more time in exploring new objects; the choice to explore new objects implicates learning of new information. The neuroanatomical substrate for this test is prefrontal cortex and hippocampus.

Open field arena $(100 * 100 * 40)$ illuminated with 40 lux dim light was used for the test.

\section{NORT was carried out in three phases}

1. Habituation - animals for habituated to the arena in two sessions of 10 minutes each with intersession interval of $4 \mathrm{hrs}$.

2. Familiarization - this session was conducted $24 \mathrm{hrs}$ after habituation session. Two identical objects were placed $90 \mathrm{~cm}$ apart, time spent by each rat in exploring the objects was recorded for 5 minutes.

3. Test - the test session was conducted with an intra-trial interval of $1 \mathrm{hr}$. Each rat was presented with a familiar object placed at the same position and an novel object at the place of the second familiar object. The time spent exploring the familiar and novel object was recorded for 5 minutes.

Following were assessed by NORT:

1. The absolute time of novel object exploration (time in seconds)

2. An exploratory preference score, i.e., time spent exploring the novel object divided by the total time spent exploring both objects $\times 100$. An exploratory preference score of $50 \%$ indicated chance performance, while higher preference scores indicate intact memory performance

3. Discrimination index (exploration of novel object (sec)-familiar object (sec)/total time (sec)

\section{Statistical analysis}

The data were analyzed by one-way analysis of variance followed by Tukey's multiple comparisons post-hoc test. Values are expressed as mean \pm standard deviation; $\mathrm{p}<0.05$ was considered statistically significant.

\section{RESULTS}

Plasma corticosterone (Fig. 1)

Fig 1 indicates the Effect of chronic unpredictable stress (CUS) and Ethanolic extract of Eclipta alba (EEEA) treatment on plasma corticosterone levels. Animals Exposed to 30 days of unpredictable stress showed increase in the levels of plasma corticosterone as compared with the control animals $(\mathrm{p}<0.05)$, when CUS animals were treated with $200 \mathrm{mg} / \mathrm{kg}$ bw of EEEA, significant decrease in the levels of plasma corticosterone was observed indicating restoration of HPA axis $(\mathrm{p}<0.05)$ and decrease in stress response. Treated group did not show any statistical changes when compared to Stress group animals $(\mathrm{p}<0.05)$

Table 1: Chronic unpredictable stress Protocol

\begin{tabular}{|c|c|c|}
\hline Day & Stressor & Duration \\
\hline $1,7,13,19,25$ & $\begin{array}{l}\text { Noise stress (100 db, white } \\
\text { noise generator) }\end{array}$ & $4 \mathrm{hrs}$ \\
\hline $2,8,14,20,26$ & $\begin{array}{l}\text { Cage isolation (small housing } \\
\text { cage for rodents) }\end{array}$ & $24 \mathrm{hrs}$ \\
\hline $3,9,15,21,27$ & Forced swim stress at $23^{\circ} \mathrm{C} \pm 1^{\circ} \mathrm{C}$ & 15 minutes \\
\hline $4,10,16,22,28$ & Immobilization stress & 4 hrs \\
\hline $5,11,17,23,29$ & Food deprivation stress & $24 \mathrm{hrs}$ \\
\hline $6,12,18,24,30$ & Overnight illuminated lights on & $12 \mathrm{hrs}$ \\
\hline
\end{tabular}

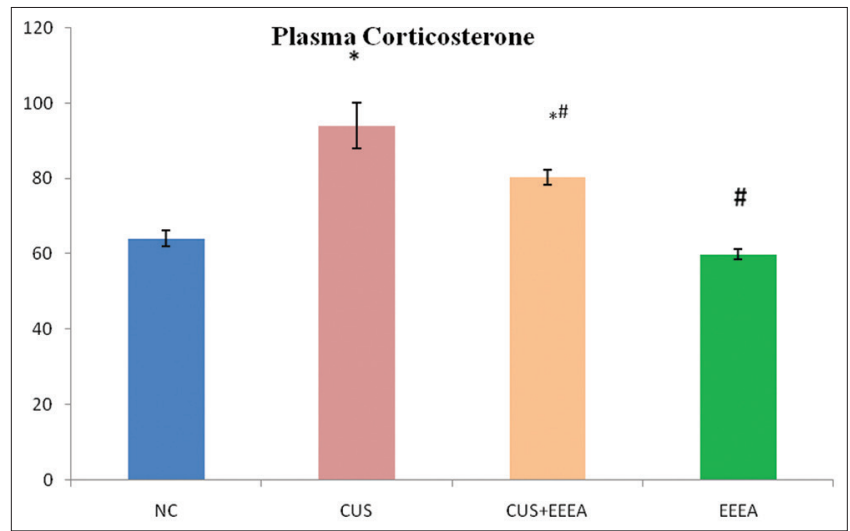

Fig. 1: Effect of chronic unpredictable stress (CUS) and ethanolic extract of Eclipta alba (EEEA) treatment on plasma corticosterone levels. All values expressed as mean \pm standard deviation. CUS exposed animals showed significantly increased plasma corticosterone as compared with control $(p<0.05)$, upon treatment of EEEA, corticosterone levels were significantly reduced $(p<0.05)$. *Compared to control and ${ }^{\#}$ Compared to CUS 


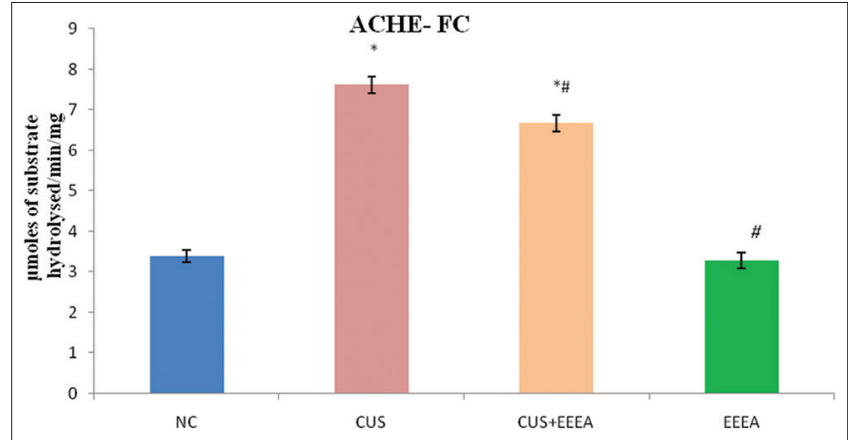

Fig. 2: Effect of chronic unpredictable stress (CUS) and ethanolic extract of Eclipta alba (EEEA) treatment on acetylcholinesterase

(AChE) activity in prefrontal cortex. All values expressed as

mean \pm standard deviation, AChE activity was significantly increased in animals exposed to 30 days of chronic unpredictable stress $(p<0.05)$, whereas significantly decreased in CUS exposed animals treated with $200 \mathrm{mg} / \mathrm{kg}$ bw EEEA $(\mathrm{p}<0.05)$. ${ }^{*}$ Compared to control and " compared to CUS

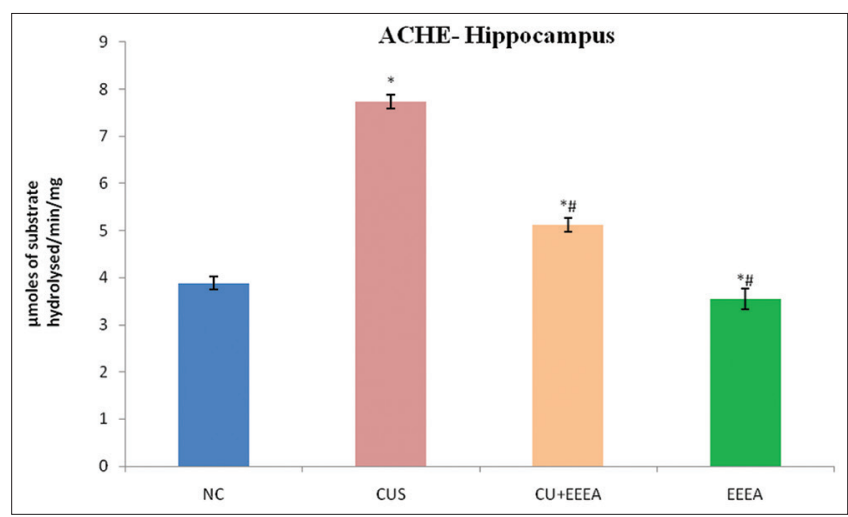

Fig. 3: Effect of chronic unpredictable stress (CUS) and ethanolic extract of Eclipta alba (EEEA) treatment on acetylcholinesterase (AChE) activity in hippocampus. All values expressed as mean \pm standard deviation, $\mathrm{AChE}$ activity was significantly increased in animals exposed to 30 days of chronic unpredictable stress $(p<0.05)$, whereas significantly decreased in CUS exposed animals treated with $200 \mathrm{mg} / \mathrm{kg}$ bw EEEA $(\mathrm{p}<0.05)$. Treated alone group did not show any difference. *Compared to control and ${ }^{\#}$ Compared to CUS

AChE activity (Figs. 2-4)

Fig 2-4 Acetyl cholinesterase activity was increased invariably in the Prefrontal cortex (Fig 2), Hippocampus (Fig 3), Corpus striatum (Fig. 4) of Stressed animals $\mathrm{p}<0.05$ indicating degradation of Acetylcholine at these regions whereas same is restored to near control values upon treatment with EEEA $200 \mathrm{mg} / \mathrm{kg}$ bw $(\mathrm{p}<0.05)$ indicating effectiveness of eclipta alba as Acetyl cholinesterase inhibitor, treatment group (EEEA alone) showed significantly lower AchE activity in the Hippocampus as compared to control $(p<0.05)$ indicating memory enhancing activity even in the normal control animals upon EEEA treatment. whereas there was no change in Corpus striatum and frontal cortex between control and treated group.

\section{NORT (Figs. 5-7)}

Exploration of Novel object is an innate behaviour of rats, when we subjected all four group rats to the NOR Task, we observed difference in the exploration behaviour.

Animals exposed to CUS spent considerably less time exploring the novel object $(\mathrm{p}<0.05)$ when compared with control and other groups.

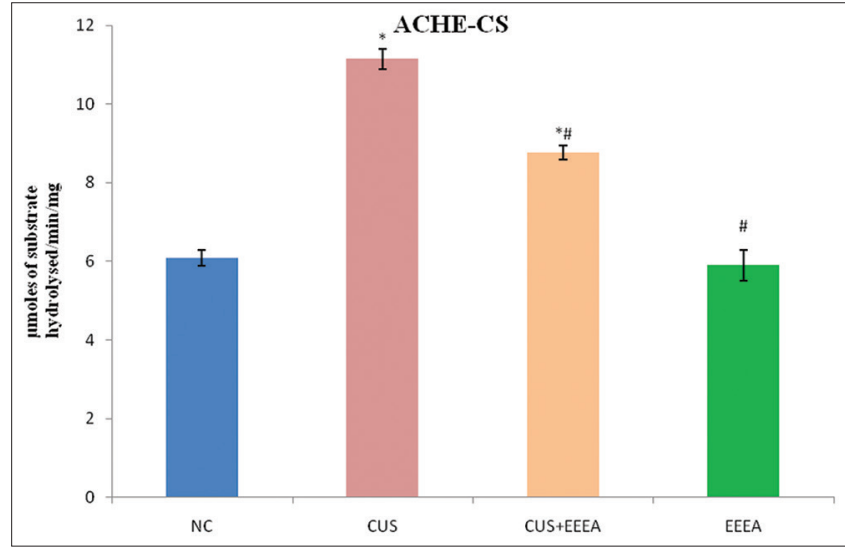

Fig. 4: Effect of chronic unpredictable stress (CUS) and ethanolic extract of Eclipta alba (EEEA) treatment on acetylcholinesterase

(AChE) activity in corpus striatum. All values expressed as mean \pm standard deviation, AChE activity was significantly increased in animals exposed to 30 days of chronic unpredictable stress $(p<0.05)$, whereas significantly decreased in CUS exposed animals treated with $200 \mathrm{mg} / \mathrm{kg}$ bw EEEA $(p<0.05)$. Treated alone group did not show any difference. *Compared to control and \#Compared to CUS

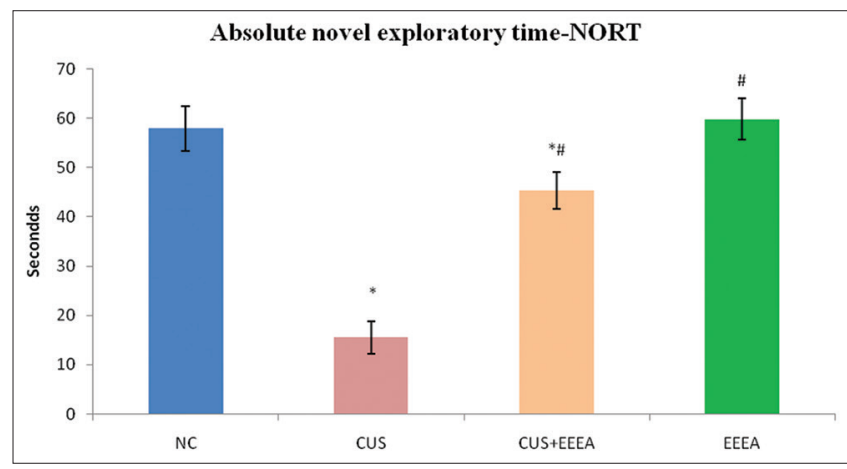

Fig. 5: Absolute novel object exploratory time is expressed in seconds. Values are expressed as mean time \pm standard deviation. The mean time of novel object exploration by chronic unpredictable stress (CUS) exposed rats are significantly lower as compared to control $(p<0.05)$, novelty behavior is restored with ethanolic extract of Eclipta alba treatment (p<0.05). *Compared to control and "Compared to CUS

Stressed rats showed less preference for Novel object (Fig 6) $p<0.05$ as compared with control and other groups and upon treatment there was significant change, Preference towards novel object was significantly more $(\mathrm{p}<0.05)$.

Discrimination Index (DI) (fig 7)allows discrimination between the novel and familiar objects, it is the difference in exploration time for familiar object, dividing this value by the total amount of exploration of the novel and familiar objects $[\mathrm{DI}=(\mathrm{TN}-\mathrm{TF}) /(\mathrm{TN}+\mathrm{TF})]$. This result can vary between +1 and -1 , where a positive score indicates more time spent with the novel object, a negative score indicates more time spent with the familiar object, and a zero score indicates a null preference. Our observation shows Negative score in case of Stressed group of animals $\mathrm{p}<0.05$, whereas positive score of other three groups shows more time spent with novel object as compared to stress group.

\section{DISCUSSION}

In the present study, male Wistar albino rats were chosen to mimic PTSD by inducing CUS. When animals exposed to different stressors 


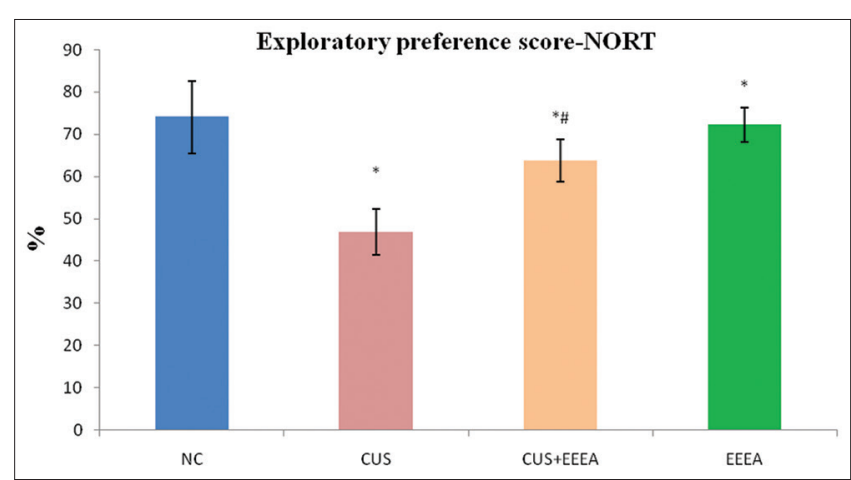

Fig. 6: Exploratory preference score is expressed in percentage, i.e., time spent exploring novel object divided by total time spent with both the objects $\times 100$, a score of $50 \%$ indicates chance performance while a higher score indicates intact memory performance. Values are expressed as a mean percentage \pm standard deviation. The exploratory preference score in chronic unpredictable stress (CUS) exposed rats was significantly lower as compared to control $(p<0.05)$, with ethanolic extract of Eclipta alba treatment significant improvement was observed $(p<0.05)$. *Compared to control and "Compared to CUS

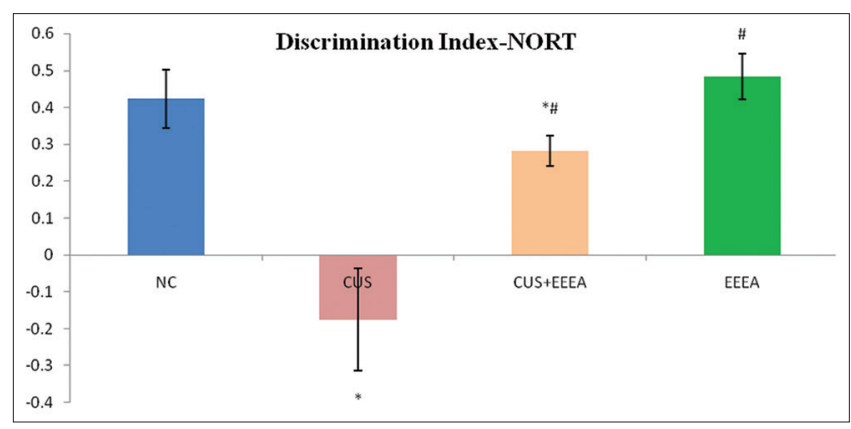

Fig. 7: Discrimination index, exploration of novel object (sec)Exploration of familiar object (sec)/total time (both objects in seconds). Values are expressed as mean percentage \pm standard deviation. Negative score of animals exposed to chronic unpredictable stress indicates more time spent with familiar object $(p<0.05)$, whereas positive scores of other groups indicate relatively more time spent with novel object

in an unpredictable manner for chronic period, causes impairment in recognition memory which may be attributed to increase in corticosteroid levels as much evidence suggests that the hippocampal abnormalities seen in chronic stress models of PTSD are due to the neurotoxic effects of corticosteroids, as recognition memory is a component of hippocampal function. In our study, a significant increase in plasma corticosterone levels in animals exposed to CUS, in accordance several studies have reported the relationship between the different types of stressors affecting memory through increased glucocorticoid levels might have implications for brain plasticity and behavioral changes following the stress in rat's hippocampus [26]. Increase in serum corticosterone level as well as hippocampal corticosterone was observed along with the memory deficit when stress was applied to male Wistar albino rats. Hippocampus, the neural component responsible for recognition memory could be affected by the increase in corticosterone levels observed in our study. when animals exposed to CUS were subjected to NOR task, it showed deficit in novel object exploring behavior, in agreement with our results other two models of PTSD, i.e., 1 week exposure to single psychosocial stress and chronic social instability combined with predator stress exposure showed impairment in NORT $[27,28]$. This vital cognitive function has been related to cholinergic neuromodulation; ACh has been demonstrated to enhance the persistent spiking of individual cortical neurons, which could provide a mechanism for active maintenance of novel information. This effect has been shown in entorhinal cortex and could also enhance encoding by enhancing long-term potentiation. ACh enhances LTP in many areas, including the hippocampus [29-31]. Principal role of AChE is the termination of nerve impulse transmission at the cholinergic synapses by rapid hydrolysis of ACh. Inhibition of AChE serves as a strategy for the treatment of memory disorders [32]. Inhibitors of AChE and butyrylcholinesterase, key enzymes involved in the degradation of neurotransmitter ACh, have been shown to function by restoring the level of ACh in the synaptic region and thus reinstate deficientcholinergic neurotransmission [33,34]. Since the discovery of cholinergic deficits in patients suffering from neurological disorders, inhibition of these enzymes is the main target in the treatment strategies [32]. Synthetic drugs used in the treatment of cognitive dysfunction associated with Alzheimer's disease and other diseases include tacrine, donepezil, and rivastigmine [35]. However, these drugs are associated with adverse effects including gastrointestinal disturbances, hepatotoxicity, and bioavailability problems [36-38]. E. alba, a dietary herb with potential anti-AChE activity [21], showed significant decrease in AChE in Frontal cortex, hippocampus, and striatum in CUS animals, which is also supported by an study where E. alba has shown to possess memory enhancing activity in scopolamine-induced amnesia model as assessed by in elevated plus maze model indicating its cognitive enhancement property [23].

In vitro study reveals that the neuroprotective effect of another herb Triphala has also found to be due to antioxidant and AChE inhibitory property [39]. Another study where four garden varieties of black tea indicated that both infusion and decoction of these tea varieties showed AChE inhibitory properties in a dose-dependent manner in mice [40].

Plants exhibiting anti-AChE activity are largely targeted for treating neuronal complications.

Our study also reveals the role of E. alba as AChE inhibitor thereby improving recognition memory in the animal model of PTSD. Findings of our study conclude that the EEEA, with rich phytochemical constituent, can emerge as a therapeutic agent useful in treating memory impairment induced by CUS.

\section{REFERENCES}

1. McEwen BS. Effects of adverse experiences for brain structure and function. Biol Psychiatry 2000;48(8):721-31.

2. Bhatia N, Maiti PP, Choudhary A, Tuli A, Masih D, Khan MM, et al. Animal models of stress. IJPSR 2011;2(5):1147-55.

3. McGuire J, Herman JP, Horn PS, Sallee FR, Sah R. Enhanced fear recall and emotional arousal in rats recovering from chronic variable stress. Physiol Behav 2010;101(4):474-82.

4. Lee DW, Chung S, Yoo HJ, Kim SJ, Woo CW, Kim ST, et al. Neurochemical changes associated with stress-induced sleep disturbance in rats: In vivo and in vitro measurements. PLoS One 2016;11(4):e0153346.

5. Ravindran R, Rathinasamy SD, Samson J, Senthilvelan M. Noisestress-induced brain neurotransmitter changes and the effect of Ocimum sanctum (Linn) treatment in albino rats. J Pharmacol Sci 2005;98(4):354-60.

6. Anuradha H, Srikumar BN, Deepti N, Shankaranarayana Rao BS, Lakshmana M. Restoration of acetylcholinesterase activity by Euphorbia hirta in discrete chronically stressed rats. Pharm Biol 2010;48(5):499-503.

7. Gilbertso MW, Shenton ME, Ciszewski A, Kasai K, Lasko NB, Orr SP, et al. Smaller hippocampal volume predicts pathologic vulnerability to psychological trauma. Nat Neurosci 2002;5(11):1242-7.

8. Bremner JD, Elzinga B, Schmahl C, Vermetten E. Structural and functional plasticity of the human brain in posttraumatic stress disorder. Prog Brain Res 2008;167:171-86.

9. Wang Z, Neylan TC, Mueller SG, Lenoci M, Truran D, Marmar CR, et al. Magnetic resonance imaging of hippocampal subfields in posttraumatic 
stress disorder. Arch Gen Psychiatry 2010;67(3):296-303.

10. Hayes JP, LaBar KS, McCarthy G, Selgrade E, Nasser J, Dolcos F. VISN Mid-Atlantic MIRECC workgroup, et al. Reduced hippocampal and amygdala activity predicts memory distortions for trauma reminders in combat-related PTSD. J Psychiatr Res 2011;45(5):660-9.

11. Samuelson KW. Post-traumatic stress disorder and declarative memory functioning: A review. Dialogues Clin Neurosci 2011;13(3):346-51.

12. Gillespie CF, Phifer J, Bradley B, Ressler KJ. Risk and resilience: Genetic and environmental influences on development of the stress response. Depress Anxiety 2009;26(11):984-92.

13. Squire LR, Wixted JT, Clark RE. Recognition memory and the medial temporal lobe: A new perspective. Nat Rev Neurosci 2007;8(11):872-83.

14. Barker GR, Warburton EC. The brain regions involved in recognition memory are also most vulnerable to stress response. J Neurosci 2011;31(29):10721-31.

15. Squire LR, Stark CE, Clark RE. The medial temporal lobe. Annu Rey Neurosci 2004;27:279-306.

16. Antunes M, Biala G. The novel object recognition memory: Neurobiology, test procedure, and its modifications. Cogn Process 2012;13(2):93-110.

17. Neeraja PV, Margaret E. Ecliptaalba (L.) Hassk: A valuable medicinal herb. Int J Curr Pharm Rev Res 2012;2(4):188-97.

18. Asolkar AV, Kakkar KK, Chakre OJ. Glossary of Indian Medicinal Plants with Active Principles. Vol. 1. New Delhi: Publication and Information Directorate (CSIR); 1992. p. 287.

19. Wagner H, Geyer B, Kiso Y, Hikino H, Rao GS. Coumestans as the main active principles of the liver drugs Eclipta alba and Wedelia calendulacea. Planta Med 1986;5:370-4

20. Mithun NM, Shashidhara S, Vivek Kumar R. Ecliptaalba (L.) A review on its phytochemical and pharmacological profile. Pharmacologyonline 2011;1:345-57.

21. Samson G, Adama H, Roland MNT, Nabèrè O, Martin T, Jeanne M, et al. Screeing of antioxidant, anti acetylcholineesterases and antifungal activitied and HPLC-MS identification of bioactive phenolics of ecliptaalbalinn. Hassk. Int J Phytomed 2012;4(4):469-76.

22. Katz RJ. Animal model of depression: Pharmacological sensitivity of a hedonic deficit. Pharmacol Biochem Behav 1982;16(6):965-8.

23. Monalisa J, Swati M, Pal A, Mishra SS. Memory enhancing activity of Eclipta alba in albino rats: A correlation with anticholinesterase activity. Int J Pharm Clin Res 2014;6(2):179-85.

24. Singh DK, Verma R. Spectrophotometric determination of corticosteroids and its application in pharmaceutical formulation. Iran J Pharmacol Ther 2008;7(1):61-5.

25. Silvers JM, Harrod SB, Mactutus CF, Booze RM. Automation of the novel object recognition task for use in adolescent rats. J Neurosci
Methods 2007;166(1):99-103.

26. Smith MA, Makino S, Kvetnansky R, Post RM. Stress and glucocorticoids affect the expression of brain-derived neurotrophic factor and neurotrophin-3 mRNAs in the hippocampus. J Neurosci $1995 ; 15: 1768-77$

27. Wang H, Zuo D, He B, Qiao F, Zhao M, Wu Y. Conditioned fear stress combined with single-prolonged stress: A new PTSD mouse model Neurosci Res 2012;73(2):142-52.

28. Zoladz PR, Conrad CD, Fleshner M, Diamond DM. Acute episodes of predator exposure in conjunction with chronic social instability as an animal model of post-traumatic stress disorder. Stress 2008;11(4):259-81.

29. Klink R, Alonso A. Muscarinic modulation of the oscillatory and repetitive firing properties of entorhinal cortex layer II neurons. J Neurophysiol 1997;77(4):1813-28.

30. Huerta PT, Lisman JE. Bidirectional synaptic plasticity induced by a single burst during cholinergic theta oscillation in CA1 in vitro. Neuron 1995;15(5):1053-63.

31. Adams SV, Winterer J, Müller W. Muscarinic signaling is required for spike-pairing induction of long-term potentiation at rat Schaffer collateral-CA1 synapses. Hippocampus 2004;14(4):413-6.

32. Rahman AU, Choudhary MI. Bioactive natural products as a potential source of new pharmacophores a theory of memory. Pure Appl Chem 2001;73(3):555-60

33. Giacobini E. Invited review: Cholinesterase inhibitors for Alzheimer's disease therapy: From tacrine to future applications. Neurochem In 1998;32(5-6):413-9.

34. Krall WJ, Sramek JJ, Cutler NR. Cholinesterase inhibitors: A therapeutic strategy for Alzheimer disease. Ann Pharmacother 1999;33(4):441-50.

35. Brenner G. Pharmacology. Philadelphia, PA: WB Saunders Company; 2000

36. Oh MH, Houghton PJ, Whang WK, Cho JH. Screening of Korean herbal medicines used to improve cognitive function for anti-cholinesterase activity. Phytomedicine 2004;11(6):544-8.

37. Schulz V. Ginkgo extract or cholinesterase inhibitors in patients with dementia: What clinical trials and guidelines fail to consider. Phytomedicine 2003;10 Suppl 4:74-9.

38. Watkins PB, Zimmerman HJ, Knapp MJ, Gracon SI, Lewis KW Hepatotoxic effects of tacrine administration in patients with Alzheimer's disease. JAMA 1994;271(13):992-8.

39. Nag G, Bratati DE. Acetylcholinesterase inhibitory activity of Terminalia chebula, Terminalia bellerica and Emblica officinalis and some phenolic compounds. Int J Pharm Pharm Sci 2011;3(3):121-4.

40. Ray S, De B. Acetylcholinesterase inhibitory properties of black tea and its polyphenolic components. Int J Pharm Pharm Sci 2012;4(3):334-7. 